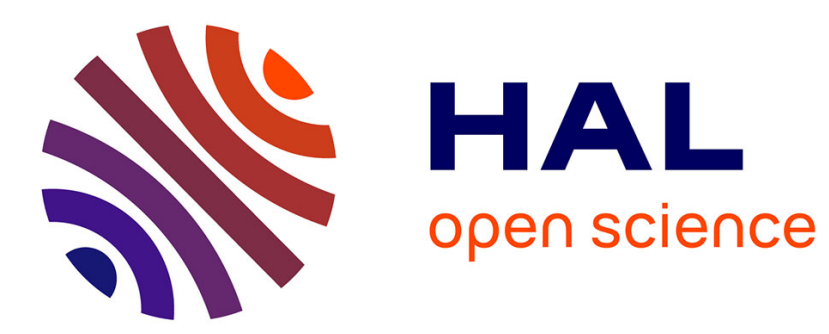

\title{
Coherent response of individual weakly confined exciton-biexciton systems
}

Jacek Kasprzak, Langbein Wolfgang

\section{To cite this version:}

Jacek Kasprzak, Langbein Wolfgang. Coherent response of individual weakly confined excitonbiexciton systems. Journal of the Optical Society of America B, 2012, 29 (7), pp.1766. 10.1364/JOSAB.29.001766 . hal-00712079

\section{HAL Id: hal-00712079 \\ https://hal.science/hal-00712079}

Submitted on 22 Sep 2021

HAL is a multi-disciplinary open access archive for the deposit and dissemination of scientific research documents, whether they are published or not. The documents may come from teaching and research institutions in France or abroad, or from public or private research centers.
L'archive ouverte pluridisciplinaire HAL, est destinée au dépôt et à la diffusion de documents scientifiques de niveau recherche, publiés ou non, émanant des établissements d'enseignement et de recherche français ou étrangers, des laboratoires publics ou privés. 


\title{
Coherent response of individual weakly confined exciton-biexciton systems
}

\author{
Jacek Kasprzak ${ }^{1,2, *}$ and Wolfgang Langbein ${ }^{1}$ \\ ${ }^{1}$ School of Physics and Astronomy, Cardiff University, The Parade, CF24 3AA Cardiff, UK \\ ${ }^{2}$ Institut Néel, CNRS/UJF, 25 av. des Martyrs, BP 16638042 Grenoble cedex 9, France \\ ${ }^{*}$ Corresponding author: jacek.kasprzak@grenoble.cnrs.fr
}

Received February 22, 2012; revised May 1, 2012; accepted May 1, 2012; posted May 3, 2012 (Doc. ID 162955); published June 25, 2012

\begin{abstract}
We investigate the coherent optical response of individual localized exciton-biexciton (X-XX) systems formed at interface fluctuations of a growth-interrupted GaAs/AlAs quantum well. We apply heterodyne spectral interferometry to perform two-dimensional four-wave mixing (FWM) spectroscopy. We retrieve the binding energy of bound and unbound XXs, as well as characterize the system in terms of biexciton-exciton dipole moment ratio and mutual FWM phase. Polarization selection rules of the FWM are determined. FWM hyperspectral imaging and autocorrelation analysis reveal the expected spatial colocalization of XXs with respect to their Xs. A value for the biexciton renormalization in a coherently coupled pair of Xs is retrieved. Our study gives insight into the coherent optical properties of an exciton-biexciton system with a confinement energy comparable to the biexciton binding energy. (C) 2012 Optical Society of America

OCIS codes: $\quad 190.4380,190.4720,190.7110,270.1670,300.6290,300.6310$.
\end{abstract}

\section{INTRODUCTION}

Various excitonic complexes [1] arise as a result of Coulomb correlations between electrons and holes in semiconductors. In particular, two excitons (Xs) form a bound biexciton [2] (XX) with a binding energy of $10 \%-50 \%$ of the exciton binding energy, typically in the milli electron volt (meV) range. The formation of excitonic complexes is particularly evident in structures with a large exciton binding energy compared to the transition linewidth induced by disorder or phonon scattering. This is typically the case in wide bandgap bulk semiconductors, like $\mathrm{CuCl}$ [3], and in semiconductor nanostructures, where due to the reduced dimensionality, Coulomb interaction is enhanced [4]. Application of nonlinear techniques, namely four-wave mixing (FWM), revealed insight into coherent response, scattering processes, and dephasing mechanisms in large ensembles of Xs and XXs in disordered GaAs quantum wells (QWs) [5-11]. The intriguing physics displayed by the coherent response of this model system is being explored [12-17], with continuously improving sophistication.

Measuring the response of individual exciton states in such ensembles gives additional insight, specifically in systems with significant disorder. Such measurements are enabled by heterodyne spectral interferometry (HSI) $[18,19]$, which allows retrieval of the complex FWM response $R^{-1,2}\left(\omega_{3}\right)$ of individual quantum systems in solids, like localized Xs in GaAs QWs. Growth interruption on both interfaces of such QWs leads to formation of monolayer islands or terraces [20,21]. The lateral size of arising natural potential fluctuations is in the $100 \mathrm{~nm}$ range, whereas the lateral confinement energy is only around $10 \mathrm{meV}$. Individual $\mathrm{X}$ states in such a natural quantum dot (QD) can exhibit a large transition dipole moment $\mu$ compared to self-assembled QDs, due to their large spatial extension $[22,23]$. Because the FWM amplitude scales as $R^{-1,2}\left(\omega_{3}\right) \propto \mu^{4}$, these Xs are well suited to explore their coherent response via HSI and were investigated intensively in the past [24-28]. Most recently [23], combining twodimensional (2D) FWM spectroscopy with hyperspectral FWM imaging, we measured coherent coupling between individual, distant Xs, and determined the mechanism to be biexcitonic renormalization.

In this paper we explore the coherent response of individual exciton-biexciton systems [29-31], weakly confined in natural QDs of a $5 \mathrm{~nm}$ GaAs QW. We use the same sample, experimental setup, and notation as in [23]. Further details concerning our methodology can be found in $[32,33]$. With HSI we measure the spectrally resolved interference $\overline{J_{D}}(\omega)$ between the reference field and the reflection from the sample detected at the frequency of the heterodyne beat $\Omega_{D}$ of the selected optical signal. Specifically, the degenerate FWM signal, $R^{-1,2} \propto \mathcal{E}_{1}^{*} \mathcal{E}_{2}^{2}$, is detected at $\Omega_{\mathrm{FWM}}=2 \Omega_{2}-\Omega_{1}$, where $\Omega_{1} /(2 \pi)=79 \mathrm{MHz}$ and $\Omega_{2} /(2 \pi)=80 \mathrm{MHz}$ are the rf upshifts of the driving fields $\mathcal{E}_{1}$ and $\mathcal{E}_{2}$, with a mutual time delay $\tau$ ranging typically up to $100 \mathrm{ps}$, positive for $\mathcal{E}_{1}$ leading. The amplitude and phase of the FWM field are obtained via spectral interferometry [34], with a reference pulse arriving prior to the FWM. The experiments were carried out at $T=10 \mathrm{~K}$ in the third-order regime, as determined by the FWM intensity dependence [28]. We use a diffraction-limited spot of around $600 \mathrm{~nm}$ FWHM and confocal detection. Due to the third-order nonlinearity in the FWM experiment, a subdiffraction spatial resolution of down to $300 \mathrm{~nm}$ is achieved. The spectral resolution allows us to isolate the different localized exciton states present in the focal area.

Using 2D FWM spectroscopy we identify bound and unbound biexciton resonances, analyze their binding energy and dipole moment ratio $\mu_{\mathrm{XX}} / \mu_{\mathrm{X}}$, and compare them with previous measurements performed on ensembles. Adjusting the polarization of the driving fields, we investigate polarization 
selection rules. We use hyperspectral FWM imaging and spectral correlation analysis to confirm the spatial colocalization of the bound XX with respect to the related X. Finally, we derive a value of the biexcitonic renormalization of a pair of spatially separated, coherently coupled Xs.

\section{RESULTS}

\section{A. Two-Dimensional Four-Wave Mixing}

2D FWM spectroscopy is performed by measuring $R^{-1,2}\left(\omega_{3}, \tau\right)$ and Fourier transforming the $\tau>0$ sequence along the delay coordinate. In Fig. 1(a) we show an example of a resulting 2D FWM diagram displaying $\left|R^{-1,2}\left(\omega_{3}, \omega_{1}\right)\right|^{2}$, measured in horizontal linear polarization configuration, denoted as $(\rightarrow, \rightarrow, \rightarrow)$, on a sample region of a low $\mathrm{X}$ density. This two-frequency representation of the FWM is suited to investigate coherent coupling between individual resonances, as it evidences which first-order frequency $\omega_{1}$ drives which corresponding third-order (FWM) frequencies $\omega_{3}$. The data are dominated by four uncoupled Xs, aligned along the diagonal $\omega_{1}=\omega_{3}$. Each of these Xs is accompanied by a weaker, spectrally narrow resonance at the same $\omega_{1}$, but at $\omega_{3}$ some $5 \mathrm{meV}$ below. These different resonances are related to transitions from the Xs to bound biexcitons, forming a second approximate diagonal that is redshifted by a biexciton binding energy, which is similar for the different Xs. Additionally, features associated with transitions from $\mathrm{X}$ to unbound biexcitons $\left(\mathrm{XX}^{*}\right)$ are also identified in the 2D FWM data. An example is given in Fig. 1(b), where an off-diagonal peak at $\omega_{3}=1699.2 \mathrm{meV}$ is blueshifted by several meV from $\mathrm{X}$ at $\omega_{1}=\omega_{3}=$ $1695.8 \mathrm{meV}$. Unlike the well-defined $\mathrm{XX}$ resonances, such $\mathrm{XX}^{*}$ signals exhibit complex line shapes, which are often distributed over $\sim 0.5 \mathrm{meV}$, consisting of a set of underlying resonances. A confirmation of the biexcitonic origin of these peaks is provided by the observation of a $\pi$-shift between the phase of $0-\mathrm{X}$ and $\mathrm{X}-\mathrm{XX}$ (or $\mathrm{X}-\mathrm{XX}^{*}$ ) transitions [23], as shown by the yellow traces in Fig. 1 . In Figs. $1(\mathrm{c})$ and $1(\bar{d})$ we present a statistical analysis of the deduced binding energies for XXs and $\mathrm{XX}^{*} \mathrm{~s}$. The $\mathrm{XX}$ states show a binding energy of $(4.9 \pm 0.2) \mathrm{meV}$. The standard deviation is only $4 \%$, which is even less than the $5 \%-7 \%$ observed in large ensembles for non-growth-interrupted QWs [11]. Instead, the repulsion energy of unbound states shows a substantial dispersion and exhibits a standard deviation of approximately $2 \mathrm{meV}$. In previous measurements on ensembles [11], the $\mathrm{XX}^{*} \mathrm{~s}$ created spectral tails over several meV on the high energy side of Xs (see Fig. 2, lower right frame, in [11] for $\tau_{12}=0$ ). The large broadening of the unbound biexciton transitions also results in their fast dephasing versus $\tau_{12}$ in ensemble measurements [35].

Via the strength of the signal measured in 2D FWM, we have inferred the oscillator strength of the $\mathrm{X}-\mathrm{XX}$ transitions with respect to $0-X$. In the third-order regime, the timeintegrated FWM amplitude of an $\mathrm{X}$ is proportional to $A_{\mathrm{X}} \propto 2 \mu_{\mathrm{X}}^{4} P_{\mathrm{X}}^{3} / \gamma_{\mathrm{X}}$, while the FWM amplitude of an $\mathrm{XX}$ reads
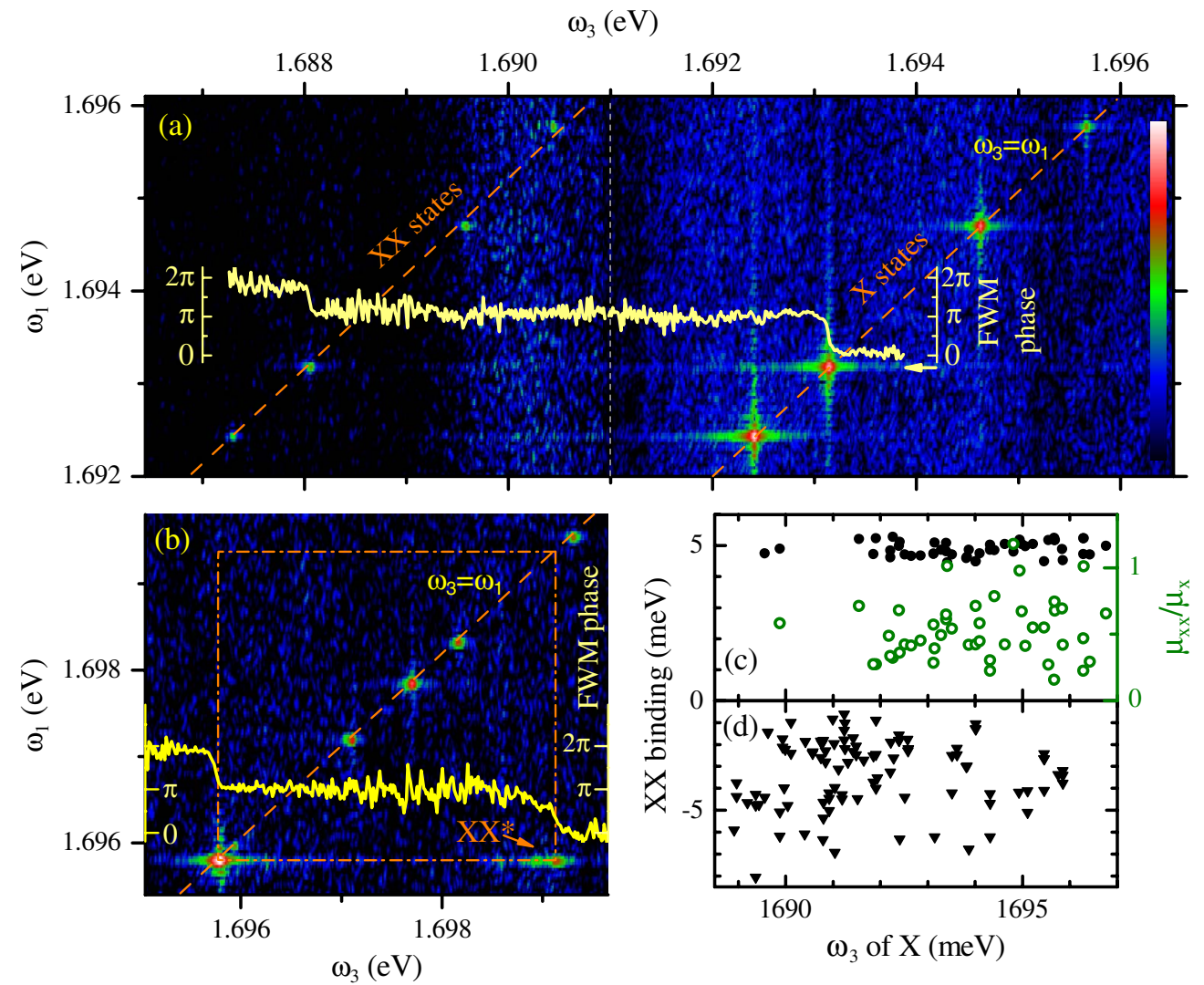

Fig. 1. (Color online) (a), (b) Two-dimensional FWM $\left|R_{\rightarrow \rightarrow \rightarrow}^{-1,2}\left(\omega_{3}, \omega_{1}\right)\right|^{2}$ showing individual Xs along with their corresponding (a) bound (XX) and (b) unbound $\left(\mathrm{XX}^{*}\right)$ biexcitons. FWM phase shifts of $\pi$ between X and XX are observed (yellow traces). In (a), logarithmic color scale over five and three orders of magnitude for $\omega_{3}>1.691 \mathrm{eV}$ and $\omega_{3}<1.691 \mathrm{eV}$ (see vertical, dashed line), respectively. Magnification by a factor of 100 for $\omega_{3}<1.691 \mathrm{eV}$ was applied to visualize XXs. In (b), logarithmic color scale over three orders of magnitude. c) Statistics of XXs' binding energy (black dots) and dipole moment ratio $\mu_{\mathrm{XX}} / \mu_{\mathrm{X}}$ (green circles), d) statistics of XX*s' binding energy (black triangles). Its negative value should be understood as a repulsion energy of a two-particle state. 
$A_{\mathrm{XX}} \propto \mu_{\mathrm{X}}^{2} \mu_{\mathrm{XX}}^{2} P_{\mathrm{X}}^{2} P_{\mathrm{XX}} / \gamma_{\mathrm{XX}}$, where $P_{\mathrm{XX}}\left(P_{\mathrm{X}}\right)$ is the excitation field (square root of the measured excitation intensity) at the $\mathrm{X}-\mathrm{XX}$ $(0-X)$ energy. Taking into consideration the spectrometer resolution of approximately $15 \mu \mathrm{eV}$, we experimentally retrieve transition linewidths for $\mathrm{X}$ and $\mathrm{XX}$ transitions $\gamma_{\mathrm{X}}$ and $\gamma_{\mathrm{XX}}$, respectively. We generally find $\gamma_{\mathrm{XX}}>\gamma_{\mathrm{X}}$. We deduce the ratio between $\mathrm{XX}$ and $\mathrm{X}$ transition dipole moments using the expression

$$
\frac{\mu_{\mathrm{Xx}}}{\mu_{\mathrm{X}}}=\sqrt{\frac{2 P_{\mathrm{X}} A_{\mathrm{Xx}} \gamma_{\mathrm{Xx}}}{P_{\mathrm{XX}} A_{\mathrm{X}} \gamma_{\mathrm{X}}}},
$$

where $A_{\mathrm{XX}}\left(A_{\mathrm{X}}\right)$ is the FWM peak amplitude of $\mathrm{XX}(\mathrm{X})$ assessed from $\left|R^{-1,2}\left(\omega_{3}, \omega_{1}\right)\right|$. The resulting distribution of $\mu_{\mathrm{XX}} / \mu_{\mathrm{X}}$ is given in Fig. $1(\mathrm{c})$.

The dipole moment of the $\mathrm{X}-\mathrm{XX}$ transitions is measured to always be weaker than the one of the corresponding $(0-X)$ transition, evidence that the XXs in these samples are in a weak confinement regime [36]. In this regime the $\mathrm{XX}$ wave function is a mixture of different $\mathrm{X}$ wave functions, with only the part of the same $\mathrm{X}$ contributing to the $\mathrm{X}-\mathrm{XX}$ dipole moment. The reported dipole moment ratios actually give a measure of the ground-state $\mathrm{X}$ wave function content within the corresponding XX. The same analysis for the unbound biexcitons reveals a large spread of $\mu_{\mathrm{XX}} / \mu_{\mathrm{X}}$. The analysis is, however, less reliable, due to broad, non-Lorentzian line shapes of the $\mathrm{X}-\mathrm{XX}^{*}$ transition that may contain several unresolved resonances.

\section{B. Polarization Dependence}

We now turn to the dependence of the FWM on the polarization of the driving fields $\mathcal{E}_{1}$ and $\mathcal{E}_{2}$. Ensemble studies on this system [11] showed polarization selection rules consistent with a four-level system of $0-\mathrm{X}-\mathrm{XX}$ considering the two optically active spin states of $\mathrm{X}$ and the pertinent polarization selection rules of the transitions. We consider here detection of the horizontal $(\rightarrow)$ linear polarization along the $[1 \overline{1} 0]$ crystal direction and first discuss the data obtained for positive delay $\tau=+1 \mathrm{ps}$ ( $\mathcal{E}_{1}$ arriving first), shown in Fig. 2. For cocircular $\left(\sigma^{+}, \sigma^{+}, \rightarrow\right)$ excitation, the FWM is induced by $\mathcal{E}_{2}$ on the $0-\mathrm{X}$ transition only (visible at $\omega_{3}=1691.8 \mathrm{meV}$ ) via the density grating created upon the consecutive arrival of $\mathcal{E}_{1}$ and $\mathcal{E}_{2}$. FWM on the X-XX transition is not observed. This indicates that the bound biexciton state is a product state of excitons of opposite circular polarization, which cannot be excited in the cocircular excitation configuration. When instead a colinear configuration $(\rightarrow, \rightarrow, \rightarrow)$ is applied, FWM on both $0-\mathrm{X}$ and $\mathrm{X}-\mathrm{XX}$ transitions (XX detected at $\omega_{3}=1686.9 \mathrm{meV}$ ) can be created via the density grating in 0 and $\mathrm{X}$. Conversely, upon crosslinear excitation $(\rightarrow, \uparrow, \rightarrow)$, the FWM is driven by $\mathcal{E}_{2}$ on the $\mathrm{X}-\mathrm{XX}$ transition only by the Raman coherence [37,38] between both $\rightarrow$ - and $\uparrow$-polarized Xs, driven by $\mathcal{E}_{1}$ and $\overline{\mathcal{E}_{2}}$, respectively.

For negative time delay $\tau=-1 \mathrm{ps}\left(\mathcal{E}_{2}\right.$ arriving first), no FWM can be observed under $\left(\sigma^{+}, \sigma^{+}, \rightarrow\right)$ excitation. In such a case, no two-photon coherence (TPC) can be excited, as the $0-\mathrm{X}$ and $\mathrm{X}-\mathrm{XX}$ transitions have opposite circular polarization. Instead, in the linear polarization basis, $0-\mathrm{X}$ and $\mathrm{X}-\mathrm{XX}$ transitions are collinearly polarized. Therefore, for $(\rightarrow, \rightarrow, \rightarrow)$ excitation, $\mathcal{E}_{2}$ can drive a TPC between the

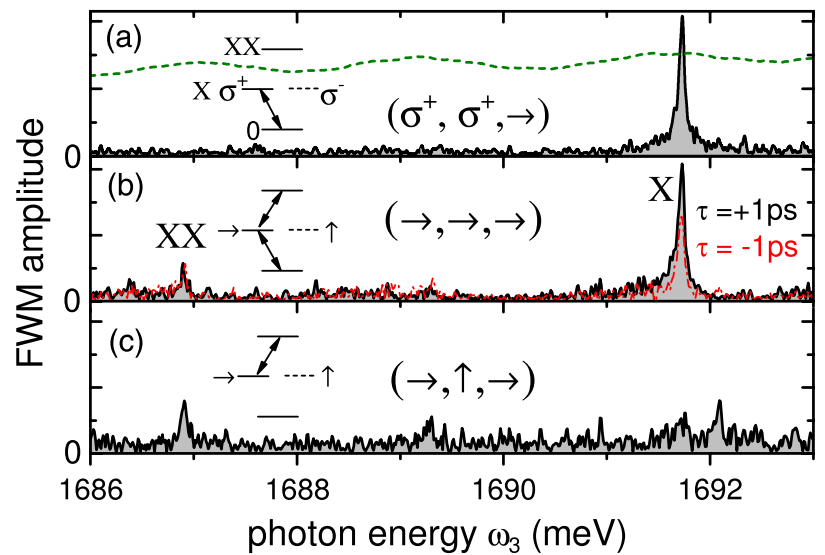

Fig. 2. (Color online) Polarization dependence and selection rules in the FWM of an individual $0-\mathrm{X}-\mathrm{XX}$ system weakly localized on a monolayer fluctuation of a $5 \mathrm{~nm}$ GaAs/AlGaAs QW. (a) $\left(\sigma^{+}, \sigma^{+}, \rightarrow\right)$ polarization configuration, $\tau=+1 \mathrm{ps}$. For negative delays no FWM is observed within the noise. The spectral amplitude of the excitation fields is given by the green dashed curve. (b) $(\rightarrow, \rightarrow, \rightarrow)$ polarization configuration producing FWM on both $0-\mathrm{X}$ and $\mathrm{X}-\mathrm{XX}$ transitions, for $\tau=+1 \mathrm{ps}$ and $\tau=-1 \mathrm{ps}$. (c) $(\rightarrow, \uparrow, \rightarrow)$ configuration. For $\tau=+1 \mathrm{ps,}$ FWM is observed at the $\mathrm{X}-\mathrm{XX}$ transition. The amplitude range in (a), (b) is three times bigger than in (c).

ground state and $\mathrm{XX}$, which upon the consecutive arrival of $\mathcal{E}_{1}$ is converted into FWM on both $0-\mathrm{X}$ and $\mathrm{X}-\mathrm{XX}$ transitions.

\section{Spatial Correlation}

It is instructive to perform real space mapping of individual $0-\mathrm{X}$ and $\mathrm{X}-\mathrm{XX}$ transitions. Such mapping has been reported on similar samples with a scanning near field optical microscope giving a high spatial resolution of $30 \mathrm{~nm}[39,40]$ observing non-resonantly excited photoluminescence. Here we explore the nonlinear coherent response of a $0-\mathrm{X}-\mathrm{XX}$ system in real space. It is realized by combining $2 \mathrm{D}$ FWM with FWM hyperspectral imaging [23]. In Fig. $\underline{3(\mathrm{a})}$ we show the FWM spectrum of the $\mathrm{X}_{4}$ resonance shown in Fig. 2 of [23]. Hyperspectral imaging was performed, and the resulting FWM spatial maps at $\omega_{3}$ frequencies corresponding to $0-\mathrm{X}(\mathrm{X}-\mathrm{XX})$ FWM transitions are presented in Fig. 3(b) [3(c)]. A colocalization of $\mathrm{XX}$ with respect to its $\mathrm{X}$ is evident. Note that the $\mathrm{X}-\mathrm{XX}$ intensity shown in Fig. 3(c) corresponds to the product of the XX and $\mathrm{X}$ wave function. Another example is shown in Figs. 3(e)-3(g), where a doublet of bound XXs arise from the same $\mathrm{X}$. Because of the weak XX signal, the colocalization is not as evident, but restricting the $\mathrm{XX}-\mathrm{X}$ signal to the range of the $\mathrm{X}$ signal it is still observable.

With the aim to analyze the statistical properties of $\mathrm{X}$ and XX spatial colocalization, we performed spectral correlation analysis. We used hyperspectral images of the FWM amplitude, $\left|R^{-1,2}\left(x, y, \omega_{3}\right)\right|$, within a data cube of $3.5 \mu \mathrm{m}, 4.5 \mu \mathrm{m}$, and $10 \mathrm{meV}$ size in the dimensions $x, y$, and $\omega_{3}$, respectively. Typical averaged FWM spectra within such ensembles are presented in Fig. A in "Supplementary Information" of [23]. We evaluate the autocorrelation

$$
\begin{aligned}
C\left(\Delta x, \Delta y, \Delta \omega_{3}\right) \\
=\frac{\left\langle\left|R^{-1,2}\left(x+\Delta x, y+\Delta y, \omega_{3}+\Delta \omega_{3}\right)\right|\left|R^{-1,2}\left(x, y, \omega_{3}\right)\right|\right\rangle_{x, y, \omega_{3}}}{\left\langle\left|R^{-1,2}\left(x, y, \omega_{3}\right)\right|\right\rangle_{x, y, \omega_{3}}^{2}} .
\end{aligned}
$$



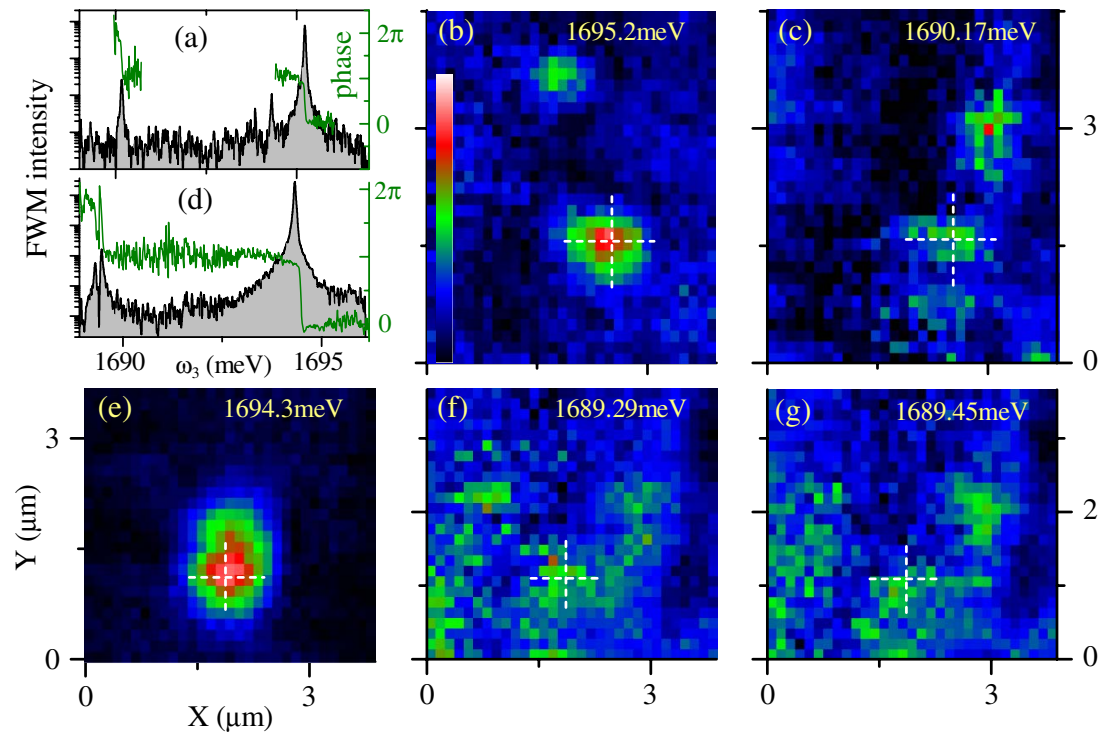

Fig. 3. (Color online) (a) FWM intensity $\left|R^{-1,2}\left(\omega_{3}\right)\right|^{2}$ (black) and phase $\arg \left[R^{-1,2}\left(\omega_{3}\right)\right]$ (green) of an individual $0-\mathrm{X}-\mathrm{XX}$ system. FWM imaging at $\tau=0.1 \mathrm{ps}(\mathrm{b})$ at the $0-\mathrm{X}$ transition energy, $\left|R^{-1,2}(x, y, 1695.2 \mathrm{meV})\right|$, and (c) at the X-XX transition energy, $\left|R^{-1,2}(x, y, 1690.17 \mathrm{meV})\right|$. The white cross indicates the X position at $(x, y)=(2.5,1.5) \mu \mathrm{m}$. (d) FWM intensity $\left|R^{-1,2}\left(\omega_{3}\right)\right|^{2}$ (black) and phase $\arg \left[R^{-1,2}\left(\omega_{3}\right)\right]($ green) showing an X-XX doublet arising from the same X. (e) Same as (b) at 0-X transition energy $\omega_{3}=1694.3 \mathrm{meV}$; (f), (g) same as (c) at X-XX transition energies $\omega_{3}=(1689.29,1689.45) \mathrm{meV}$. The white cross indicates the $\mathrm{X}$ position at $(x, y)=(1.9,1.1) \mu \mathrm{m}$. Linear color scale from 0 to $(0.3,0.9,10,1,1)$ arbitrary units in (b), (c), (e), (f), (g), respectively.

To investigate the distance dependence, we use polar coordinates $C\left(\Delta r \cos \phi, \Delta r \sin \phi, \Delta \omega_{3}\right)$ and average over the azimuthal angle $\phi=0 \ldots 2 \pi$, yielding $\bar{C}\left(\Delta r, \Delta \omega_{3}\right)$. Subtracting the spectral autocorrelation at large distances $(4 \mu \mathrm{m})$, we plot the background-corrected spectral autocorrelation $\Delta \bar{C}\left(\Delta \omega_{3}\right)=\bar{C}\left(0, \Delta \omega_{3}\right)-\bar{C}\left(4 \mu \mathrm{m}, \Delta \omega_{3}\right)$ in Fig. 4 . We observe that $\Delta \bar{C}\left(\Delta \omega_{3}\right)$ shows a peak at $\Delta \omega_{3}$, corresponding to the $\mathrm{XX}$ binding energy. However, due to the rather weak XX FWM signal, it is not very pronounced. Furthermore, $\Delta \bar{C}\left(\Delta \omega_{3}\right)$ shows features related to the correlation of $\mathrm{X}$ resonances, indicating a typical spectral separation of colocalized excitons of $4 \mathrm{meV}$ in the $5 \mathrm{~nm} \mathrm{QW}$ and $3 \mathrm{meV}$ in the $7 \mathrm{~nm} \mathrm{QW}$ (as also directly inferred via FWM hyperspectral imaging).

\section{Biexcitonic Renormalization}

In [23] we have shown that a biexcitonic renormalization energy $(\delta)$ enables coherent coupling between a pair of Xs. Here we detail the related analysis to retrieve $\delta$. In Fig. $\underline{5(\mathrm{a})}$ we
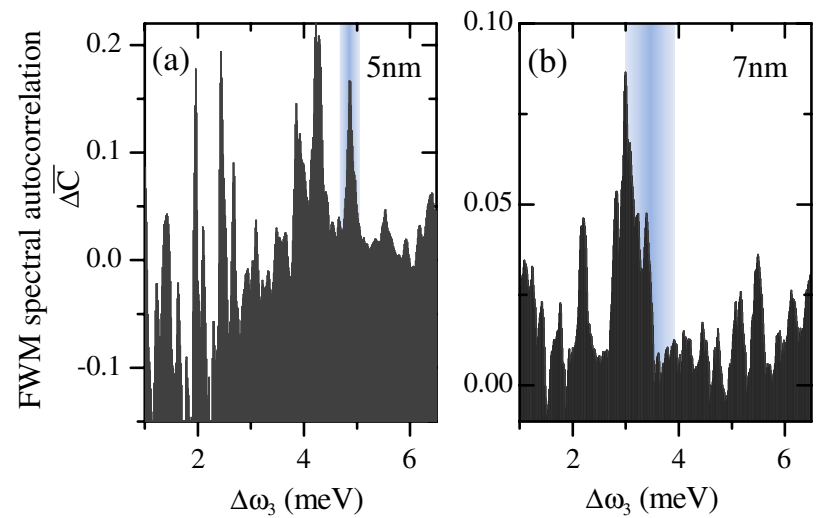

Fig. 4. (Color online) FWM hyperspectral background-corrected autocorrelation, $\Delta \bar{C}\left(\Delta \omega_{3}\right)$. Analysis performed on (a) a $5 \mathrm{~nm}$ and (b) a $7 \mathrm{~nm} \mathrm{GaAs} / \mathrm{AlGaAs}$ disordered QW. Blue shaded regions indicate measured $\mathrm{XX}$ binding energies of $(4.9 \pm 0.2) \mathrm{meV}$ (a) and $(3.5 \pm 0.9) \mathrm{meV}(\mathrm{b})$. show an example of 2D FWM data, $\left|R^{-1,2}\left(\omega_{3}, \omega_{1}\right)\right|^{2}$, measured on a sample region of a higher $\mathrm{X}$ density than the one probed in Fig. 1. Coherent coupling between X resonances at 1688.37 and $16 \overline{9} 1.16 \mathrm{meV}$ is detected [23]; off-diagonal peaks show that the first-order frequencies mutually drive the third-order frequencies of both Xs. To retrieve the value of $\delta$, we first plot spectral cuts of the real and imaginary part of

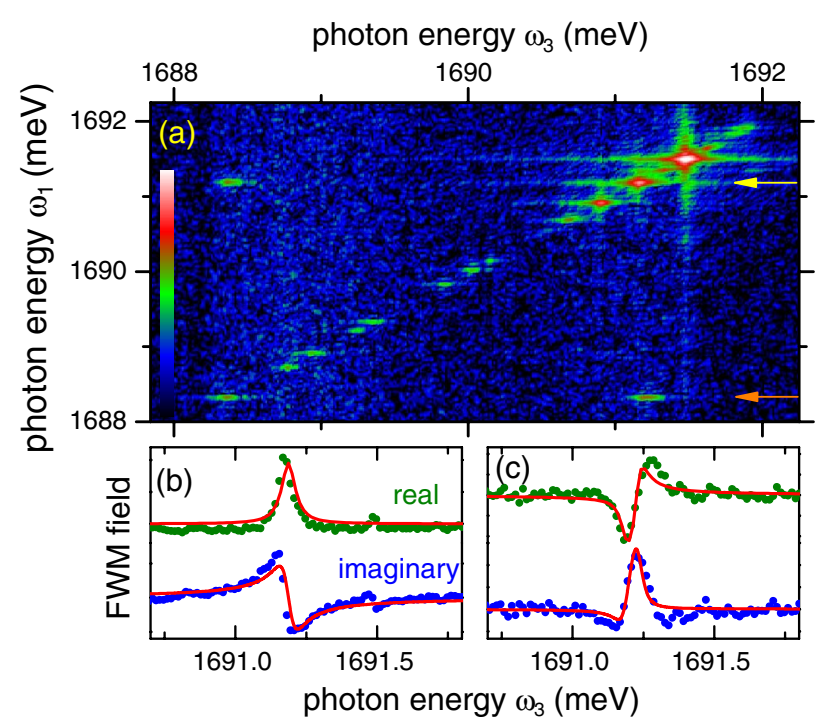

Fig. 5. (Color online) Retrieval of biexcitonic renormalization $\delta$ providing coherent coupling between individual Xs in a $5 \mathrm{~nm} \mathrm{QW}$. (a) $\left|R_{\rightarrow \rightarrow, \rightarrow}^{-1,}\left(\omega_{3}, \omega_{1}\right)\right|^{2}$ revealing coherently coupled Xs at $\omega_{3}=$ $1688.37 \mathrm{meV}$ and $\omega_{3}=1691.16 \mathrm{meV}$. Logarithmic color scale over 4.5 orders of magnitude. (b) FWM field $R_{\rightarrow \rightarrow \rightarrow}^{-1,2}\left(\omega_{3}, \omega_{1}\right)$ in real and imaginary parts at $\omega_{1}=1691.16 \mathrm{meV}$ (along the yellow arrow). The $\mathrm{X}$ resonance at $\omega_{3}=1691.16 \mathrm{meV}$ is fitted by a single complex Lorentzian (red traces). (c) Same as (b), but at $\omega_{1}=1688.37 \mathrm{meV}$ (along the orange arrow). The off-diagonal signal is modeled (red traces) with a double complex Lorentzian of the $0-X$ and $\mathrm{X}-\mathrm{XX}$ transitions (see text), yielding $\delta=(31-4 i) \mu \mathrm{eV}$. 
$R^{-1,2}\left(\omega_{3}, 1691.16 \mathrm{meV}\right)$ and fit the diagonal resonance at $\omega_{3}=$ $1691.16 \mathrm{meV}$ with a single complex Lorentzian

$$
L_{\mathrm{X}}(\omega)=\frac{A_{\mathrm{X}} \exp (i \varphi)}{i \gamma_{\mathrm{X}}+\left(\omega-\omega_{\mathrm{X}}\right)}
$$

as shown in Fig. 5b. Even though not resolved in the 2D FWM intensity spectra [Fig. 5(a)], off-diagonal signals consist of two out-of-phase resonances for the biexcitonic coupling. This has been shown by inspecting the FWM phase profiles, revealing $2 \pi$ shifts across off-diagonals [23], and is also observed here. One component of this doublet is due to the $0-\mathrm{X}$ transition described by Eq. (3), whereas the second component is due to the $\mathrm{X}-\mathrm{XX}$ transition, which has a complex energy shift of $\delta$ compared to the $0-\mathrm{X}$ transition. We therefore fit a double complex Lorentzian

$$
L_{\mathrm{off}}(\omega)=\frac{A_{\mathrm{X}} \exp (i \varphi)}{i \gamma_{\mathrm{X}}+\left(\omega-\omega_{\mathrm{X}}\right)}-\frac{A_{\mathrm{XX}} \exp (i \varphi)}{i \gamma_{\mathrm{XX}}+\left(\omega-\omega_{\mathrm{XX}}\right)},
$$

containing the off-diagonal peak at $\omega_{1}=1688.37 \mathrm{meV}$ [see Fig. 5(c)]. The latter is performed while keeping fixed previously extracted excitonic parameters $A_{\mathrm{X}}, \gamma_{\mathrm{X}}$, and $\omega_{\mathrm{X}}$. Finally, $\delta$ is calculated as $\delta=\omega_{\mathrm{XX}}-\omega_{\mathrm{X}}-i\left(\gamma_{\mathrm{XX}}-\gamma_{\mathrm{X}}\right)=$ $(31-4 i) \mu \mathrm{eV}$ within an error of a few $\mu \mathrm{eV}$. The interexciton coupling is therefore due to a real biexcitonic repulsion, whereas the dissipative coupling (exciton-exciton scattering) is negligible. This example demonstrates the sensitivity of the HSI technique to quantitatively characterize weak coherent coupling within a small ensemble of Xs.

\section{SUMMARY}

In summary, we investigated the coherent response of individual $0-\mathrm{X}-\mathrm{XX}$ systems weakly confined in monolayer fluctuations of a disordered narrow GaAs QW. Bound and unbound XX transitions were detected in 2D FWM spectroscopy and characterized in terms of their binding energy and oscillator strength ratio $\mu_{\mathrm{XX}} / \mu_{\mathrm{X}}$. This analysis provides a measure of the projection of the $\mathrm{XX}$ wave function onto the $\mathrm{X}$ product state. The measured small values of $\mu_{\mathrm{XX}} / \mu_{\mathrm{X}}$ show that XX contains significant admixtures of other $X$ states. We have presented examples of FWM polarization control within such 0-X-XX systems. Furthermore, employing hyperspectral FWM imaging, we have demonstrated spatial colocalization of $\mathrm{X}$ and XX states. Finally, we have retrieved the value of biexcitonic renormalization, enabling coherent coupling within a pair of individual Xs.

\section{ACKNOWLEDGMENTS}

We acknowledge support by the European Commission under Marie Curie Fellowship "CUSMEQ" and UK EPSRC under Contract No. EP/D025303/1.

\section{REFERENCES}

1. M. A. Lampert, "Mobile and immobile effective-mass-particle complexes in nonmetallic solids," Phys. Rev. Lett. 1, 450-453 (1958).

2. J. R. Haynes, "Experimental observation of the excitonic molecule,” Phys. Rev. Lett. 17, 860-862 (1966)

3. H. Akiyama, T. Kuga, M. Matsuoka, and M. Kuwata-Gonokami, "Radiative decay and phonon scattering of biexcitons in $\mathrm{CuCl}$," Phys. Rev. B 42, 5621-5625 (1990).
4. U. Woggon, K. Hild, F. Gindele, W. Langbein, M. Hetterich, M. Grün, and C. Klingshirn, "Huge binding energy of localized biexcitons in CdS/ZnS quantum structures," Phys. Rev. B 61, 12632-12635 (2000).

5. T. F. Albrecht, K. Bott, T. Meier, A. Schulze, M. Koch, S. T. Cundiff, J. Feldmann, W. Stolz, P. Thomas, S. W. Koch, and E. O. Göbel, "Disorder mediated biexcitonic beats in semiconductor quantum wells," Phys. Rev. B 54, 4436-4439 (1996).

6. A. Euteneuer, J. Möbius, R. Rettig, E. J. Mayer, M. Hofmann, W. Stolz, E. O. Göbel, and W. W. Rühle, "Biexcitonic binding energies in the transition regime from three- to two-dimensional semiconductors,” Phys. Rev. B 56, R10028-R10031 (1997).

7. W. Langbein and J. M. Hvam, "Localized biexcitons in quasi-2D and quasi-3D systems,” Phys. Stat. Sol. B 206, 111-118 (1998).

8. W. Langbein and J. M. Hvam, "Localization-enhanced biexciton binding in semiconductors," Phys. Rev. B 59, 15405-15408 (1999)

9. W. Langbein and J. M. Hvam, "Dephasing in the quasi twodimensional exciton-biexciton system," Phys. Rev. B 61, 1692-1695 (2000).

10. W. Langbein, T. Meier, S. Koch, and J. Hvam, "Spectral signatures of $\chi^{(5)}$ processes in four-wave mixing of homogeneously broadened excitons,” J. Opt. Soc. Am. B 18, 1318-1325 (2001).

11. W. Langbein and J. M. Hvam, "Biexcitonic bound and continuum states of homogeneously and inhomogeneously broadened exciton resonances," Phys. Stat. Sol. A 190, 167-174 (2002).

12. S. R. Bolton, U. Neukirch, L. J. Sham, D. S. Chemla, and V. M. Axt, "Demonstration of sixth-order coulomb correlations in a semiconductor single quantum well," Phys. Rev. Lett. 85, 2002-2005 (2000).

13. K. W. Stone, K. Gundogdu, D. B. Turner, X. Li, S. T. Cundiff, and K. A. Nelson, "Two-quantum 2D FT electronic spectroscopy of biexcitons in GaAs quantum wells," Science 324, 1169-1173 (2009).

14. A. D. Bristow, D. Karaiskaj, X. Dai, R. P. Mirin, and S. T. Cundiff, "Polarization dependence of semiconductor exciton and biexciton contributions to phase-resolved optical two-dimensional Fourier-transform spectra,” Phys. Rev. B 79, 161305 (2009).

15. D. B. Turner and K. A. Nelson, "Coherent measurements of high-order electronic correlations in quantum wells," Nature 466, 1089-1092 (2010).

16. D. Karaiskaj, A. D. Bristow, L. Yang, X. Dai, R. P. Mirin, S. Mukamel, and S. T. Cundiff, "Two-quantum many-body coherences in two-dimensional Fourier-transform spectra of exciton resonances in semiconductor quantum wells," Phys. Rev. Lett. 104, 117401 (2010).

17. D. B. Turner, P. Wen, D. H. Arias, and K. A. Nelson, "Coherent two-exciton dynamics measured using two-quantum rephasing two-dimensional electronic spectroscopy," Phys. Rev. B 84, 165321 (2011).

18. W. Langbein and B. Patton, "Heterodyne spectral interferometry for multidimensional nonlinear spectroscopy of individual quantum systems," Opt. Lett. 31, 1151-1153 (2006).

19. W. Langbein and B. Patton, "Transient coherent nonlinear spectroscopy of single quantum dots,” J. Phys. Condens. Matter 19, 295203 (2007).

20. D. Gammon, B. V. Shanabrook, and D. S. Katzer, "Interfaces in GaAs/AlAs quantum well structures," Appl. Phys. Lett. 57, 2710-2712 (1990).

21. K. Leosson, J. R. Jensen, W. Langbein, and J. M. Hvam, "Exciton localization and interface roughness in growth-interrupted GaAs/AlAs quantum wells," Phys. Rev. B 61, 10322-10329 (2000).

22. V. Savona and W. Langbein, "Realistic heterointerface model for excitonic states in growth-interrupted GaAs quantum wells," Phys. Rev. B 74, 075311 (2006).

23. J. Kasprzak, B. Patton, V. Savona, and W. Langbein, "Coherent coupling between distant excitons revealed by two-dimensional nonlinear hyperspectral imaging," Nat. Photon. 5, 57-63 (2011).

24. W. Langbein and B. Patton, "Microscopic measurement of photon echo formation in groups of individual excitonic transitions,” Phys. Rev. Lett. 95, 017403 (2005).

25. B. Patton, U. Woggon, and W. Langbein, "Coherent control and polarization readout of individual excitonic states," Phys. Rev. Lett. 95, 266401 (2005). 
26. Y. Wu, X. Li, L. M. Duan, D. G. Steel, and D. Gammon, "Density matrix tomography through sequential coherent optical rotations of an exciton qubit in a single quantum dot," Phys. Rev. Lett. 96, 087402 (2006).

27. J. Kasprzak and W. Langbein, "Vectorial four-wave mixing field dynamics from individual excitonic transitions," Phys. Rev. B 78, 041103R (2008).

28. J. Kasprzak and W. Langbein, "Four-wave mixing from individual excitons: Intensity dependence and imaging," Phys. Stat. Sol. B 246, 820-823 (2009).

29. E. T. Batteh, J. Cheng, G. Chen, D. G. Steel, D. Gammon, D. S. Katzer, and D. Park, "Coherent nonlinear optical spectroscopy of fluctuation quantum dots: evidence for coupling between quantum dots," Phys. Rev. B 71, 155327 (2005).

30. G. Chen, T. H. Stievater, E. T. Batteh, X. Li, D. G. Steel, D. Gammon, D. S. Katzer, D. Park, and L. J. Sham, "Biexciton quantum coherence in a single quantum dot," Phys. Rev. Lett. 88, 117901 (2002).

31. X. Li, Y. Wu, X. Xu, D. G. Steel, and D. Gammon, "Transient nonlinear optical spectroscopy studies involving biexciton coherence in single quantum dots," Phys. Rev. B 73, 153304 (2006).

32. P. Borri and W. Langbein, in Semiconductor Quantum Bits, F. Henneberger and O. Benson, eds. (Pan Stanford, 2009), pp. 269-320.

33. W. Langbein, "Coherent optical spectroscopy of semiconductor nanostructures," Riv. Nuovo Cimento 33, 255-312(2010).
34. L. Lepetit, G. Chériaux, and M. Joffre, "Linear techniques of phase measurement by femtosecond spectral interferometry for applications in spectroscopy," J. Opt. Soc. Am. B 12, 2467-2474 (1995).

35. W. Langbein, J. M. Hvam, M. Umlauff, H. Kalt, B. Jobst, and D. Hommel, "Binding-energy distribution and dephasing of localized biexcitons," Phys. Rev. B 55, R7383-R7386 (1997).

36. A. V. Filinov, C. Riva, F. M. Peeters, Y. E. Lozovik, and M. Bonitz, "Influence of well-width fluctuations on the binding energy of excitons, charged excitons and biexcitons in GaAs-based quantum wells," Phys. Rev. B 70, 035323 (2004).

37. K. B. Ferrio and D. G. Steel, "Raman quantum beats of interacting excitons," Phys. Rev. Lett. 80, 786-789 (1998).

38. X. Li, Y. Wu, D. G. Steel, D. Gammon, and L. J. Sham, "Raman coherence beats from the entangled state involving polarized excitons in single quantum dots," Phys. Rev. B 70, 195330 (2004).

39. K. Matsuda, T. Saiki, S. Nomura, M. Mihara, Y. Aoyagi, S. Nair, and T. Takagahara, "Near-field optical mapping of exciton wave functions in a GaAs quantum dot," Phys. Rev. Lett. 91, 177401 (2003).

40. Y. Sugimoto, T. Saiki, and S. Nomura, "Visualization of weak confinement potentials by near-field optical imaging spectroscopy of exciton and biexciton in a single quantum dot," Appl. Phys. Lett. 93, 083116 (2008). 